\title{
LOCALLY UNIFORMLY CONVEX BANACH SPACES
}

\author{
BY \\ A. R. LOVAGLIA
}

Introduction. In $1936 \mathrm{~J}$. A. Clarkson [2, p. 396] introduced the notion of uniform convexity of the norm in a Banach space. Stated in geometric terms, a norm is uniformly convex if whenever the midpoint of a variable chord in the unit sphere of the space approaches the boundary of the sphere, the length of the chord approaches zero. Clarkson [2, p. 403] has shown that for $p>1$ the spaces $L_{p}$ and $l_{p}$ are uniformly convex.

It is the object of this paper to consider a weaker type of convexity, which we shall call local uniform convexity. Geometrically this differs from uniform convexity in that it is required that one end point of the variable chord remain fixed. In section I we prove a general theorem on the product of locally uniformly convex Banach spaces and with the aid of this theorem we establish that the two notions are actually different. Section II is devoted to the investigation of the relationship between local uniform convexity and strong differentiability of the norm. In section III we investigate conditions for isomorphism of a Banach space with a locally uniformly convex space.

In his paper entitled Uniform convexity [5, p. 745] M. M. Day defines the notion of local uniform convexity near a point as follows: If $\left\|b_{0}\right\|=1, B$ is said to be locally uniformly convex near $b_{0}$ if there is a sphere about $b_{0}$ in which the condition for uniform convexity holds. Geometrically this differs from uniform convexity in that the variable chord in the unit sphere is contained in a sphere about some point $b_{0}$, whereas local uniform convexity (l.u.c.) as defined in definition 0.2 below requires only that one end point of the chord remain fixed. In Theorem 1 of his paper [5, p. 746] Day proves that if a space $B$ is locally uniformly convex near a point $b_{0}$, then $B$ is isomorphic to a uniformly convex space. Hence local uniform convexity near a point $b_{0}$ implies isomorphism of the space $B$ with an l.u.c. space. However the example following Theorem 1.1 below shows that there exist l.u.c. spaces not isomorphic to any uniformly convex space, which shows that the notion of 1.u.c. and Day's notion of local uniform convexity near a point are essentially different.

This paper is part of a thesis submitted in partial fulfillment of the requirements for the $\mathrm{Ph}$.D. degree at the University of California. I wish to thank Professor R. C. James for the time and assistance he gave in the preparation of this paper.

We now give several definitions.

Definition 0.1. A normed linear space is uniformly convex if, and only if, given $\epsilon>0$, there exists $\delta(\epsilon)>0$, such that

Presented to the Society, May 1, 1954; received by the editors October 9, 1953. 


$$
\frac{\|x+y\|}{2} \leqq 1-\delta(\epsilon) \text { whenever }\|x-y\| \geqq \epsilon, \text { and }\|x\|=\|y\|=1 .
$$

DEFINITION 0.2. A normed linear space is locally uniformly convex (1.u.c.) if, and only it, given $\epsilon>0$ and an element $x$ with $\|x\|=1$, there exists $\delta(\epsilon, x)>0$ such that

$$
\frac{\|x+y\|}{2} \leqq 1-\delta(\epsilon, x) \text { whenever }\|x-y\| \geqq \epsilon \text { and }\|y\|=1 .
$$

Definition 0.3. A normed linear space is strictly convex if, and only if, $\|x+y\|=\|x\|+\|y\|$ implies $x=t \cdot y, t>0$, whenever $x \neq 0$ and $y \neq 0$.

It is clear from the definitions that uniform convexity implies local uniform convexity, and local uniform convexity implies strict convexity.

Definition 0.4. The norms \|\| and \|\|$_{1}$ are equivalent if, and only if, for any sequence $\left\{x_{n}\right\}$

$$
\lim _{n \rightarrow \infty}\left\|x_{n}\right\|=0 \text { if, and only if, } \quad \lim _{n \rightarrow \infty}\left\|x_{n}\right\|_{1}=0 .
$$

It can be shown that a necessary and sufficient condition for \|\| and \|\|$_{1}$ to be equivalent is that there exist numbers $a, b$ with $0<a \leqq b<\infty$ such that

$$
a \cdot\|x\| \leqq\|x\|_{1} \leqq b \cdot\|x\|
$$

for all $x$.

Definition 0.5. The normed linear spaces $B$ and $B_{1}$ are said to be $i s o$ morphic if, and only if, there exists a one-to-one linear transformation of $B$ onto all of $B_{1}$.

If $U$ is this transformation, then $U^{-1}$ is also linear [1, p. 180]. It follows then that there exist numbers $a, b$ with $0<a \leqq b<\infty$ such that, for all $x$ in $B$, $a \cdot\|x\| \leqq\|U(x)\|_{1} \leqq b \cdot\|x\|$, where \|\|$_{1}$ is the norm in $B_{1}$. Thus we can define a new norm in $B$, say \|\|$^{\prime}$, where $\|x\|^{\prime}=\|U(x)\|_{1}$, which is equivalent to the original norm. And conversely if a norm, \|\|$_{1}$, equivalent to the norm \|\| , in $B$ can be defined over the linear space $B$, then the normed linear space $B_{1}$, which is the linear space $B$ with norm \|\|$_{1}$, is isomorphic to the normed linear space $B$.

We now state Minkowski's inequality: If $p>1$ and $\left\{a_{n}\right\},\left\{b_{n}\right\}$ are sequences of real numbers for which $\sum_{n=1}^{\infty}\left|a_{n}\right|^{p}$ and $\sum_{n=1}^{\infty}\left|b_{n}\right|^{p}$ are finite, then $\left(\sum_{n=1}^{\infty}\left|a_{n}+b_{n}\right|^{p}\right)^{1 / p} \leqq\left(\sum_{n=1}^{\infty}\left|a_{n}\right|^{p}\right)^{1 / p}+\left(\sum_{n=1}^{\infty}\left|b_{n}\right|^{p}\right)^{1 / p}$, and equality holds if and only if, for some $t>0, a_{n}=t \cdot b_{n}$ for all $n$.

I. The product of locally uniformly convex Banach spaces. Let $\left\{B_{n}\right\}$ be a sequence of 1.u.c. Banach spaces. Denote by \|\|$_{n}$ the norm in $B_{n}$. Let $P_{2}\left(B_{n}\right)$ be the space of all sequences, $x=\left\{x^{n}\right\}, x^{n}$ in $B_{n}$, for which $\sum_{n=1}^{\infty}\left\|x^{n}\right\|_{n}^{2}$ is convergent. Let \|\| be the norm in $P_{2}\left(B_{n}\right)$ where $\|x\|=\left(\sum_{n=1}^{\infty}\left\|x^{n}\right\|_{n}^{2}\right)^{1 / 2}$. It is readily verified that $P_{2}\left(B_{n}\right)$ is a Banach space. 
TheOREM 1.1. $P_{2}\left(B_{n}\right)$ is l.u.c.

Proof. Let

$$
\begin{array}{r}
x=\left(x^{1}, \cdots, x^{k}, x^{k+1} \cdots\right) ; \quad x_{k}=\left(0, \cdots, 0, x^{k+1}, x^{k+2}, \cdots\right), \quad k \geqq 1 ; \\
y_{p}=\left(y_{p}^{1}, \cdots, y_{p}^{k}, y_{p}^{k+1}, \cdots\right) ; \quad y_{p, k}=\left(0, \cdots, 0, y_{p}^{k+1}, y_{p}^{k+2}, \cdots\right), \\
p=1,2, \cdots .
\end{array}
$$

Let $\|x\|=\left\|y_{p}\right\|=1$ for all $p$. Thus $x$ is an element and $\left\{y_{p}\right\}$ is a sequence of elements on the unit sphere of $P_{2}\left(B_{n}\right)$. Now suppose that $\lim _{p \rightarrow \infty}\left\|x+y_{p}\right\|=2$. According to the definition of l.u.c. the theorem will be proved if we can show that

$$
\lim _{p \rightarrow \infty}\left\|x-y_{p}\right\|=0 .
$$

Using the properties of the norm and the Minkowski inequality we obtain the following inequalities:

$$
\begin{aligned}
\left\|x+y_{p}\right\| & =\left(\sum_{n=1}^{\infty}\left\|x^{n}+y_{p}^{n}\right\|_{n}^{2}\right)^{1 / 2} \\
& =\left(\sum_{n=1}^{k}\left\|x^{n}+y_{p}^{n}\right\|_{n}^{2}+\sum_{n=k+1}^{\infty}\left\|x^{n}+y_{p}^{n}\right\|_{n}^{2}\right)^{1 / 2} \\
& =\left(\sum_{n=1}^{k}\left\|x^{n}+y_{p}^{n}\right\|_{n}^{2}+\left\|x_{k}+y_{p, k}\right\|^{2}\right)^{1 / 2} \\
& \leqq\left(\sum_{n=1}^{k}\left(\left\|x^{n}\right\|_{n}+\left\|y_{p}^{n}\right\|_{n}\right)^{2}+\left(\left\|x_{k}\right\|+\left\|y_{p, k}\right\|\right)^{2}\right)^{1 / 2} \\
& \leqq\left(\sum_{n=1}^{k}\left\|x^{n}\right\|_{n}^{2}+\left\|x_{k}\right\|^{2}\right)^{1 / 2}+\left(\sum_{n=1}^{k}\left\|y_{p}^{n}\right\|_{n}^{2}+\left\|y_{p, k}\right\|^{2}\right)^{1 / 2} \\
& =\|x\|+\left\|y_{p}\right\|=2 .
\end{aligned}
$$

Thus $\left\|x+y_{p}\right\| \leqq\left(\sum_{n=1}^{k}\left(\left\|x^{n}\right\|_{n}+\left\|y_{p}^{n}\right\|_{n}\right)^{2}+\left(\left\|x_{k}\right\|+\left\|y_{p, k}\right\|\right)^{2}\right)^{1 / 2} \leqq 2$. Since by hypothesis $\lim _{p \rightarrow \infty}\left\|x+y_{p}\right\|=2$, we have

$$
\lim _{p \rightarrow \infty}\left(\sum_{n=1}^{k}\left(\left\|x^{n}\right\|_{n}+\left\|y_{p}^{n}\right\|_{n}\right)^{2}+\left(\left\|x_{k}\right\|+\left\|y_{p, k}\right\|\right)^{2}\right)^{1 / 2}=2 .
$$

Now, for each $n,\left\{\left\|y_{p}^{n}\right\|_{n}\right\}$ is a bounded sequence of real numbers, since $\left\|y_{p}\right\|^{2}=1$, and hence $\left\|y_{p}^{n}\right\|_{n}^{2} \leqq 1$ for all $p$. Hence by diagonalizing we can determine a sequence of $p$ 's for which there exists $\lim _{p \rightarrow \infty}\left\|y_{p}^{n}\right\|_{n}=a_{n}$, for each $n$. Also

$$
1=\left\|y_{p}\right\|^{2}=\sum_{n=1}^{k}\left\|y_{p}^{n}\right\|_{n}^{2}+\left\|y_{p, k}\right\|^{2} \text {. }
$$


Therefore $\lim _{p \rightarrow \infty}\left\|y_{p, k}\right\|^{2}=1-\sum_{n=1}^{k} a_{n}^{2}=b_{k}^{2}$. Hence using the above results,

$$
\begin{aligned}
\lim _{p \rightarrow \infty}\left(\sum_{n=1}^{k}\left(\left\|x^{n}\right\|_{n}+\left\|y_{p}^{n}\right\|_{n}\right)^{2}+\right. & \left.\left(\left\|x_{k}\right\|+\left\|y_{p, k}\right\|\right)^{2}\right)^{1 / 2} \\
& =\left(\sum_{n=1}^{k}\left(\left\|x^{n}\right\|_{n}+a_{n}\right)^{2}+\left(\left\|x_{k}\right\|+b_{k}\right)^{2}\right)^{1 / 2}=.2 .
\end{aligned}
$$

But $\left(\sum_{n=1}^{k}\left\|x^{n}\right\|_{n}^{2}+\left\|x_{k}\right\|^{2}\right)^{1 / 2}+\left(\sum_{n=1}^{k} a_{n}^{2}+b_{k}^{2}\right)^{1 / 2}=2$. Equating the left-hand members and employing the Minkowski inequality and the fact that $\|x\|=1$ and $k$ was arbitrary, we obtain $a_{n}=\left\|x^{n}\right\|_{n}$ for $n=1,2, \cdots$, and $b_{k}=\left\|x_{k}\right\|$. Thus we have shown that, assuming $\lim _{p \rightarrow \infty}\left\|x+y_{p}\right\|=2$, it follows that $\lim _{p \rightarrow \infty}\left\|y_{p}^{n}\right\|_{n}=\left\|x^{n}\right\|_{n}$ and $\lim _{p \rightarrow \infty}\left\|y_{p, k}\right\|=\left\|x_{k}\right\|$.

We now show that $\lim _{p \rightarrow \infty}\left\|x-y_{p}\right\|=0$, by showing that the negation of this leads to a contradiction. To this end suppose there exists a subsequence of $p$ 's for which $\left\|x-y_{p}\right\| \geqq r>0$. Then

$$
\begin{aligned}
0<r \leqq\left\|x-y_{p}\right\| & =\left(\sum_{n=1}^{k}\left\|x^{n}-y_{p}^{n}\right\|_{n}^{2}+\sum_{n=k+1}^{\infty}\left\|x^{n}-y_{p}^{n}\right\|_{n}^{2}\right)^{1 / 2} \\
& =\left(\sum_{n=1}^{k}\left\|x^{n}-y_{p}^{n}\right\|_{n}^{2}+\left\|x_{k}-y_{p, k}\right\|^{2}\right)^{1 / 2} \\
& \leqq\left(\sum_{n=1}^{k}\left\|x^{n}-y_{p}^{n}\right\|_{n}^{2}\right)^{1 / 2}+\left\|x_{k}-y_{p, k}\right\| \\
& \leqq\left(\sum_{n=1}^{k}\left\|x^{n}-y_{p}^{n}\right\|_{n}^{2}\right)^{1 / 2}+\left\|x_{k}\right\|+\left\|y_{p, k}\right\| .
\end{aligned}
$$

Therefore $\left(\sum_{n=1}^{k}\left\|x^{n}-y_{p}^{n}\right\|_{n}^{2}\right)^{1 / 2} \geqq r-\left(\left\|x_{k}\right\|+\left\|y_{p, k}\right\|\right)$. By virtue of the fact that $\lim _{p \rightarrow \infty}\left\|y_{p, k}\right\|=\left\|x_{k}\right\|$ and $\lim _{k \rightarrow \infty}\left\|x_{k}\right\|=0$, there exist $k$ and $p_{0}$ such that for all $p \geqq p_{0},\left\|x_{k}\right\|+\left\|y_{p, k}\right\|<r$. Consequently for this choice of $k$ and all $p \geqq p_{0}$,

$$
\left(\sum_{n=1}^{k}\left\|x^{n}-y_{p}^{n}\right\|_{n}^{2}\right)^{1 / 2} \geqq s>0 .
$$

Hence there exists an $n_{0}, 1 \leqq n_{0} \leqq k$, and a subsequence of $p$ 's for which $\left\|x^{n_{0}}-y_{p}^{n_{0}}\right\|_{n_{0}} \geqq t>0$. Now $\left.\left\|x^{n_{0}}+y_{p}^{n_{0}}\right\|\right|_{n_{0}} \leqq\left\|x^{n_{0}}\right\|_{n_{0}}+\left\|y_{p}^{n_{0}}\right\|_{n_{0}}$ and $\lim _{p \rightarrow \infty}\left(\left\|x^{n_{0}}\right\|_{n_{0}}\right.$ $\left.+\left\|y_{p}^{n_{0}}\right\|_{n_{0}}\right)=2\left\|x^{n_{0}}\right\|_{n_{n}}$. Therefore $\left\|x^{n_{0}}\right\|_{n_{0}} \neq 0$. And so there exists a subsequence of $p$ 's for which $\left\|y_{p}^{n_{0}}\right\|_{n_{0}} \neq 0$. Now

$$
\begin{aligned}
\liminf _{p \rightarrow \infty}\left\|\frac{x^{n_{0}}}{\left\|x^{n_{0}}\right\|_{n_{0}}}-\frac{y_{p}^{n_{0}}}{\left\|y_{p}^{n_{0}}\right\|_{n_{0}}}\right\|_{n_{0}} & =\liminf _{p \rightarrow \infty}\left\|\frac{x^{n_{0}}}{\left\|x^{n_{0}}\right\|_{n_{0}}}-\frac{y_{p}^{n_{0}}}{\left\|x^{n_{0}}\right\|_{n_{0}}}\right\|_{n_{0}} \\
& \geqq \frac{t}{\left\|x^{n_{0}}\right\|_{n_{0}}} \geqq t>0,
\end{aligned}
$$


since $\lim _{p \rightarrow \infty}\left\|y_{p}^{n_{0}}\right\|_{n_{0}}=\left\|x^{n_{0}}\right\|_{n_{0}}$ and $\left\|x^{n_{0}}\right\|_{n_{0}} \leqq 1$. Therefore, since $B_{n_{0}}$ is 1.u.c. we have

$$
\limsup _{p \rightarrow \infty}\left\|\frac{x^{n_{0}}}{\left\|x^{n_{0}}\right\|_{n_{0}}}+\frac{y_{p}^{n_{0}}}{\left\|y_{p}^{n_{0}}\right\|_{n_{0}}}\right\|_{n_{0}} \leqq 2-\delta_{n_{0}}, \quad \text { where } \delta_{n_{0}}=\delta_{n_{0}}\left(t, x^{n_{0}}\right)>0 .
$$

Therefore $\lim \sup _{p \rightarrow \infty}\left\|x^{n_{0}}+y_{p}^{n_{0}}\right\|_{n_{0}} \leqq\left(2-\delta_{n_{0}}\right) \cdot\left\|x^{n_{0}}\right\|_{n_{0}}$. Now $\left\|x+y_{p}\right\| \leqq\left(\sum_{n=1}^{k} \| x^{n}\right.$ $\left.+y_{p}^{n} \|_{n}^{2}\right)^{1 / 2}+\left\|x_{k}\right\|+\left\|y_{p, k}\right\|$. Therefore

$$
\begin{aligned}
\limsup _{p \rightarrow \infty}\left\|x+y_{p}\right\| & \leqq\left(\sum_{n=1}^{k} \limsup _{p \rightarrow \infty}\left\|x^{n}-y_{p}^{n}\right\|_{n}^{2}\right)^{1 / 2}+2\left\|x_{k}\right\| \\
& \leqq\left(\left(2-\delta_{n_{0}}\right)^{2} \cdot\left\|x^{n_{0}}\right\|^{2}+\sum_{n=1}^{k} 2^{2}\left\|x^{n}\right\|_{n}^{2}\right)^{1 / 2}+2\left\|x_{k}\right\| .
\end{aligned}
$$

Letting $k$ approach infinity, we have

$$
\begin{aligned}
\limsup _{p \rightarrow \infty}\left\|x+y_{p}\right\| & \leqq\left(\left(2-\delta_{n_{0}}\right)^{2} \cdot\left\|x^{n_{0}}\right\|_{n_{0}}^{2}+\sum_{n=1, n \neq n_{0}}^{\infty} 2^{2}\left\|x^{n}\right\|_{n}^{2}\right)^{1 / 2} \\
& <\left(\sum_{n=1}^{\infty} 2^{2}\left\|x^{n}\right\|_{n}^{2}\right)^{1 / 2}=2 .
\end{aligned}
$$

But this contradicts the assumption that $\lim _{p \rightarrow \infty}\left\|x+y_{p}\right\|=2$. Consequently $\lim _{p \rightarrow \infty}\left\|x-y_{p}\right\|=0$, and $P_{2}\left(B_{n}\right)$ is 1.u.c. Q.E.D.

We now give an example of a Banach space which is l.u.c. but is not isomorphic to any uniformly convex space.

Consider the sequence $\left\{B_{n}\right\}, n=2,3, \cdots$, of Banach spaces where $B_{n}$ is the $n$-dimensional space of points

$$
b_{n}=\left(b_{n 1}, \cdots, b_{n n}\right), \text { and }\left\|b_{n}\right\|_{n}=\left(\sum_{j=1}^{n}\left|b_{n i}\right|^{n}\right)^{1 / n} .
$$

Then each $B_{n}$ is uniformly convex [2, p. 403] and hence is l.u.c. Define $P_{2}\left(B_{n}\right)$ as in Theorem 1.1. Then, by Theorem 1.1, $P_{2}\left(B_{n}\right)$ is a l.u.c. Banach space. However, by an argument used by M. M. Day [3, p. 316] it can be shown that $P_{2}\left(B_{n}\right)$ is not isomorphic to any uniformly convex space.

To illustrate the fact that l.u.c. is stronger than strict convexity, consider the following example. Let $C$ denote the space of real continuous functions $f$ on the closed interval $[0,1]$ with $\|f\|=\max _{0 \leqq t \leqq 1}|f(t)|$. Let $\left\{t_{n}\right\}$ be a dense sequence of points in $[0,1]$ which does not include 0 . Define a new norm \|\|$_{1}$ on $C$ as follows:

$$
\|f\|_{1}=\left(\|f\|^{2}+\sum_{n=1}^{\infty} \frac{1}{2^{2 n}} \cdot\left|f\left(t_{n}\right)\right|^{2}\right)^{1 / 2}
$$


Then $\|f\| \leqq\|f\|_{1} \leqq\left(2 / 3^{1 / 2}\right)\|f\|$ for all $f$ in $C$. Hence, \|\|$_{1}$ is equivalent to \|\| . Moreover \|\|$_{1}$ is strictly convex [2, p. 413]. We shall show that \|\|$_{1}$ is not l.u.c.

Consider the function $g$, where $g(t)=3^{1 / 2} / 2$, for all $t$ in $[0,1]$, and the sequence of functions $\left\{f_{p}\right\}(p=1,2,3, \cdots)$, where

$$
f_{p}(t)=\left\{\begin{array}{l}
\frac{3^{1 / 2}}{2} \text { if } \frac{1}{p} \leqq t \leqq 1 \\
\frac{3^{1 / 2}}{2} \cdot p t \text { if } 0 \leqq t \leqq \frac{1}{p} .
\end{array}\right.
$$

Then $\|g\|_{1}=1, \quad \lim _{p \rightarrow \infty}\left\|f_{p}\right\|_{1}=1, \quad$ and $\quad \lim _{p \rightarrow \infty}\left\|f_{p}+g\right\|_{1}=2$. But $\left\|f_{p}-g\right\|$ $=\max _{0 \leqq t \leqq 1}\left|f_{p}(t)-g(t)\right|=3^{1 / 2} / 2$, for all $p$. Therefore, $\left\|f_{p}-g\right\|_{1} \geqq 3^{1 / 2} / 2$ for all $p$. Hence \|\|$_{1}$ is not l.u.c. [Definition 0.2].

The l.u.c. product of l.u.c. Banach spaces. We now give a generalization of Theorem 1.1. Let $B$ be a Banach space of sequences of real numbers such that if $y=\left\{y^{n}\right\}$ is in $B$ and $x=\left\{x^{n}\right\}$ is a sequence of real numbers with $\left|x^{n}\right| \leqq\left|y^{n}\right|$ for each $n$, then $x$ is in $B$. Let $N$ be a norm on $B$ with the following properties:

1. $B$ with norm $N$ is l.u.c.

2. $N$ is strictly increasing in each component; that is, if $\left|x^{j}\right|<\left|y^{j}\right|$, then $N\left(x^{1}, \cdots, x^{j}, \cdots\right)<N\left(x^{1}, \cdots, y^{j}, x^{j+1}, \cdots\right)$.

3. If $x=\left\{x^{n}\right\}$ is in $B$ and $x_{k}=\left(0, \cdots, 0, x^{k+1}, x^{k+2}, \cdots\right)$, then

$$
\lim _{\boldsymbol{k} \rightarrow \infty} N\left(x_{k}\right)=0 \text {. }
$$

Let $P=P_{N}\left(B_{n}\right), n=1,2,3, \cdots$, where $B_{n}$ is a Banach space for each $n$, and $x$ is in $P$ if, and only if, $x=\left\{x^{n}\right\}, x^{n}$ in $B_{n}$, and $\left\{\left\|x^{n}\right\|_{n}\right\}$ is in $B$. ( \|\|$_{n}$ is the norm in $B_{n}$.) Let the norm of $x$ in $P$ be $\|x\|=N\left(\left\{\left\|x^{n}\right\|_{n}\right\}\right)$. The space $P$ with this norm shall be called the l.u.c. product of Banach spaces.

The proof that $P$ is a Banach space presents no difficulties, so we omit it. In the case where $B_{n}$ is l.u.c. for each $n$ we have

THeOREм 1.2. The l.u.c. product of l.u.c. Banach spaces is l.u.c.

The proof of Theorem 1.2 is analogous to the proof of Theorem 1.1, so it will not be given here.

One might ask whether there exists a theorem analogous to Theorem 1.2 for uniformly convex Banach spaces. Specifically one might consider the product of uniformly convex Banach spaces and take the norm in the product space to satisfy all the conditions of Theorem 1.2 and in addition have the property of being uniformly convex for a Banach space of sequences of real numbers. However, the example immediately following Theorem 1.1 shows that such a theorem does not exist, since $l_{2}$ is uniformly convex and the norm in $l_{2}$, being $\left(\sum_{n=1}^{\infty}\left|x^{n}\right|^{2}\right)^{1 / 2}$, satisfies all the conditions of Theorem 1.2.

We do however have the following result on the product of uniformly 
convex spaces due to M. M. Day. If $\left\{B_{n}\right\}$ is a sequence of uniformly convex Banach spaces, then given $\epsilon>0$, there exists for each $B_{n}$ a positive number $\delta_{n}(\epsilon)$ such that $x^{n}, y^{n}$ in $B_{n},\left\|x^{n}\right\|_{n}=\left\|y^{n}\right\|_{n}=1$, and $\left\|x^{n}-y^{n}\right\|_{n}>\epsilon$ implies $\left\|x^{n}+y^{n}\right\|_{n}<2\left(1-\delta_{n}(\epsilon)\right)$. The sequence $\left\{B_{n}\right\}$ is said to have a common modulus of convexity $[4$, p. 504] if there is one function $\delta(\epsilon)>0$ which can be used here in place of all $\delta_{n}(\epsilon)$. Define $P_{p}\left(B_{n}\right), 1<p<\infty$, as in Theorem 1.1 above, except now let $B_{n}$ be uniformly convex for each $n=1,2, \cdots$ and for $x=\left\{x^{n}\right\}$ in $P_{p}\left(B_{n}\right),\|x\|=\left(\sum_{n=1}^{\infty}\left\|x^{n}\right\|_{n}^{p}\right)^{1 / p}<\infty$. Day $[4$, p. 505] has proved the following theorem: $B=P_{p}\left(B_{n}\right)$ is uniformly convex if and only if the $B_{n}$ have a common modulus of convexity.

II. Differentiability of the norm in a Banach space. We shall now consider some relationships between local uniform convexity of the norm and differentiability of the norm in a Banach space. In what follows, $B$ will denote an arbitrary Banach space.

Definition 2.1. The sequence $\left\{x_{n}\right\}, x_{n}$ in $B,\left\|x_{n}\right\|=1$, is an extremal sequence for $f$ in $\bar{B}$ if, and only if, $\lim _{n \rightarrow \infty} f\left(x_{n}\right)$ exists and $\left|\lim _{n \rightarrow \infty} f\left(x_{n}\right)\right|=\|f\|$, where $\bar{B}$ is the adjoint space of $B$.

Definition 2.2. The norm in $B$ is weakly differentiable at $x_{0}$ in $B$ if, and only if, $\lim _{h \rightarrow 0}\left(\left\|x_{0}+h x\right\|-\left\|x_{0}\right\|\right) / h$ exists for every $x$ in $B$. If the convergence to the limit is uniform in the unit sphere, $\|x\| \leqq 1$, of $B$, the norm is said to be stronglydifferentiable at $x_{0}$. If the norm in $B$ is everywhere strongly differentiable and the convergence to the limit is uniform with respect to $x_{0}$ and $x$ when $\left\|x_{0}\right\|=1$ and $\|x\| \leqq 1$, then the norm is called uniformly strongly differentiable. $644]$.

The following theorem and corollaries were given by V. Smulian $[8, \mathrm{p}$.

TheOREM 2.1. Let $f_{0}$ be in $\bar{B},\left\|f_{0}\right\|=1$. Then, for strong differentiability of the norm in $\bar{B}$ at $f_{0}$, it is necessary and sufficient (n.a.s.) that the following condition be satisfied:

For every extremal sequence $\left\{x_{n}\right\}$ of $f_{0}$ and every $f$ in $\bar{B}$ with $\|f\| \leqq 1$, the sequence $\left\{f\left(x_{n}\right) \cdot f_{0}\left(x_{n}\right)\right\}$ converges uniformly to a limit not depending upon the choice of extremal sequence, $\left\{x_{n}\right\}$.

CoROllaRy 1. For the strong differentiability of the norm in $\bar{B}$ at the point $f_{0},\left\|f_{0}\right\|=1$, it is n.a.s. that the following condition be satisfied:

From $\lim _{n \rightarrow \infty} f_{0}\left(x_{n}\right)=\left\|f_{0}\right\|,\left\|x_{n}\right\|=1$, it follows that $\lim _{m, n \rightarrow \infty}\left\|x_{m}-x_{n}\right\|=0$.

CoROllary 2. For the strong differentiability of the norm in $B$ at the point $x_{0},\left\|x_{0}\right\|=1$, it is n.a.s. that the following condition be satisfied:

From $\lim _{n \rightarrow \infty} f_{n}\left(x_{0}\right)=\left\|x_{0}\right\|, f_{n}$ in $\bar{B},\left\|f_{n}\right\|=1$, follows $\lim _{m, n \rightarrow \infty}\left\|f_{m}-f_{n}\right\|=0$.

Smulian $[8$, p. 648] has shown that the norm in $B$ is uniformly strongly differentiable if, and only if, the adjoint space $\bar{B}$ is uniformly convex. We make use of Theorem 2.1 and Corollaries 1 and 2 to establish similar relation- 
ships between strong differentiability of the norm and local uniform convexity.

THEOREM 2.2. If $B$ is l.u.c. and linear functionals attain their maximum on the unit sphere in $B$, then the norm is strongly differentiable in the adjoint space, $\bar{B}$.

Proof. Let $f_{0}$ be in $\bar{B},\left\|f_{0}\right\|=1$. Let $\lim _{n \rightarrow \infty} f_{0}\left(x_{n}\right)=\left\|f_{0}\right\|, x_{n}$ in $B,\left\|x_{n}\right\|=1$. We must show that $\lim _{m, n \rightarrow \infty}\left\|x_{m}-x_{n}\right\|=0$ (Corollary 1 ). By hypothesis there exists an $x_{0}$ in $B$ such that $\left\|x_{0}\right\|=1$ and $\left\|f_{0}\right\|=f_{0}\left(x_{0}\right)=1$. (1) Now suppose $\left\|x_{n}-x_{0}\right\|$ does not approach zero as $n$ tends to infinity. Then there exists a number $r>0$ and a subsequence of $n$ 's such that, for each $n,\left\|x_{n}-x_{0}\right\| \geqq r>0$. Choose a subsequence of this sequence such that $1 \geqq f_{0}\left(x_{n}\right) \geqq 1-1 / n$, for each $n$. Then $2 \geqq\left\|x_{n}+x_{0}\right\|=\left\|f_{0}\right\| \cdot\left\|x_{n}+x_{0}\right\| \geqq\left|f_{0}\left(x_{n}+x_{0}\right)\right|=\left|f_{0}\left(x_{n}\right)+f_{0}\left(x_{0}\right)\right| \geqq 1$ $-1 / n+1=2-1 / n$ and $\lim _{n \rightarrow \infty}\left\|x_{n}+x_{0}\right\|=2$. But this contradicts the hypothesis that $B$ is 1.u.c. Therefore $\lim _{n \rightarrow \infty}\left\|x_{n}-x_{0}\right\|=0$ in (1), and so

$$
\lim _{m, n \rightarrow \infty}\left\|x_{m}-x_{n}\right\|=0 \text {. }
$$

Theorem 2.3. If $\bar{B}$ is l.u.c., then the norm is strongly differentiable in $B$.

Proof. Let $x_{0}$ be in $B,\left\|x_{0}\right\|=1$. Let $\lim _{n \rightarrow \infty} f_{n}\left(x_{0}\right)=\left\|x_{0}\right\|, f_{n}$ in $\bar{B}$ and $\left\|f_{n}\right\|=1$. We must show that $\lim _{m, n \rightarrow \infty}\left\|f_{m}-f_{n}\right\|=0$ (Corollary 2). There exists an $f_{0}$ in $\bar{B}$ such that $f_{0}\left(x_{0}\right)=\left\|x_{0}\right\|$ and $\left\|f_{0}\right\|=1\left[1\right.$, p. 55]. (1) Suppose $\left\|f_{n}-f_{0}\right\|$ does not approach zero as $n$ tends to infinity. Then there exists a number $r>0$ and a subsequence of $\left\{f_{n}\right\}$ such that $\left\|f_{n}-f_{0}\right\| \geqq r$. Choose a subsequence of this sequence such that $1 \geqq f_{n}\left(x_{0}\right) \geqq 1-1 / n$. Then

$$
2 \geqq\left\|f_{n}+f_{0}\right\|=\sup _{\|x\| \geqq 1}\left|f_{n}(x)+f_{0}(x)\right| \geqq\left|f_{n}\left(x_{0}\right)+f_{0}\left(x_{0}\right)\right| \geqq 1-1 / n+1=2-1 / n,
$$

and $\lim _{n \rightarrow \infty}\left\|f_{n}+f_{0}\right\|=2$. But this contradicts the hypothesis that $\bar{B}$ is 1.u.c. Therefore $\lim _{n \rightarrow \infty}\left\|f_{n}-f_{0}\right\|=0$ in (1). And so $\lim _{m, n \rightarrow \infty}\left\|f_{m}-f_{n}\right\|=0$. Q.E.D.

Definition 2.3. $B$ is weakly l.u.c. if, and only if, $\lim _{n \rightarrow \infty}\left\|x_{n}+x_{0}\right\|=2$, $\left\|x_{n}\right\|=\left\|x_{0}\right\|=1$, implies $\lim _{n \rightarrow \infty} f_{0}\left(x_{n}\right)=\left\|x_{0}\right\|$, where $f_{0}$ is in $\bar{B},\left\|f_{0}\right\|=1$, and $f_{0}\left(x_{0}\right)=\left\|x_{0}\right\|$.

It is easily seen that if $B$ is l.u.c., then $B$ is weakly l.u.c.

THEOREм 2.4. If $B$ is weakly l.u.c. and the norm in $\bar{B}$ is strongly differentiable, then $B$ is l.u.c.

Proof. Let $\lim _{n \rightarrow \infty}\left\|x_{n}+x_{0}\right\|=2 ; x_{0}, x_{n}$ in $B,\left\|x_{0}\right\|=\left\|x_{n}\right\|=1$. Let $f_{0}$ be in $\bar{B}$ with $\left\|f_{0}\right\|=1$ and $f_{0}\left(x_{0}\right)=\left\|x_{0}\right\|$. Since $B$ is weakly 1.u.c., $\lim _{n \rightarrow \infty} f_{0}\left(x_{n}\right)=\left\|x_{0}\right\|$ $=\left\|f_{0}\right\|$. Since the norm in $\bar{B}$ is strongly differentiable, we have

$$
\lim _{m, n \rightarrow \infty}\left\|x_{m}-x_{n}\right\|=0
$$


[Corollary 1, Theorem 2.1]. Therefore there exists $y_{0}$ in $B$ with $\left\|y_{0}\right\|=1$ and $\lim _{n \rightarrow \infty}\left\|x_{n}-y_{0}\right\|=0$. Hence, $\lim _{n \rightarrow \infty} f_{0}\left(x_{n}\right)=f_{0}\left(y_{0}\right)$. Therefore $f_{0}\left(x_{0}\right)=f_{0}\left(y_{0}\right)$ $=\left\|f_{0}\right\|$. But if the norm in $\bar{B}$ is strongly differentiable, then every linear functional attains its maximum at exactly one point. Therefore $x_{0}=y_{0}$, and $\lim _{n \rightarrow \infty}\left\|x_{n}-x_{0}\right\|=0$. That is, $B$ is 1.u.c. Q.E.D.

Combining Theorem 2.2 and Theorem 2.4 we have

THEOREM 2.5. If linear functionals attain their maximum on the unit sphere of $B$, then a n.a.s. condition that $B$ be l.u.c. is that $B$ be weakly l.u.c. and the norm in $\bar{B}$ be strongly differentiable.

Definition 2.4. $\bar{B}$ is weakly l.u.c. as a set of linear functionals if, and only if, $f_{0}$ in $\bar{B},\left\|f_{0}\right\|=1, x_{0}$ in $B,\left\|x_{0}\right\|=1, f_{0}\left(x_{0}\right)=\left\|x_{0}\right\|$, and $\lim _{n \rightarrow \infty}\left\|f_{n}+f_{0}\right\|=2$, $\left\|f_{n}\right\|=1$, imply that $\lim _{n \rightarrow \infty} f_{n}\left(x_{0}\right)=\left\|x_{0}\right\|$.

It is evident that if $\bar{B}$ is l.u.c., then $\bar{B}$ is weakly l.u.c. as a set of linear functionals.

It has been shown by V. Smulian [7, p. 92] that if linear functionals attain their maximum on the unit sphere of $B$, then for $\bar{B}$ to be strictly convex it is n.a.s. that the norm be weakly differentiable in $B$. This will be used in the following theorem, as well as the evident fact that if $\bar{B}$ is strictly convex, then $x_{0}$ in $B,\left\|x_{0}\right\|=1$, and $f, g$ in $\bar{B}$ with $f\left(x_{0}\right)=\|f\|, g\left(x_{0}\right)=\|g\|$ implies that $f=c \cdot g$ where $c>0$.

THEOREM 2.6. If

(1) the norm in $B$ is strongly differentiable, and

(2) linear functionals attain their maximum on the unit sphere of $B$, and

(3) $\bar{B}$ is weakly l.u.c. as a set of linear functionals, then $\bar{B}$ is l.u.c.

Proof. Let $f_{0}$ be in $\bar{B},\left\|f_{0}\right\|=1$, and $\lim _{n \rightarrow \infty}\left\|f_{n}+f_{0}\right\|=2,\left\|f_{n}\right\|=1$. By (2) there exists an $x_{0}$ in $B$ with $\left\|x_{0}\right\|=1$ and $f_{0}\left(x_{0}\right)=\left\|f_{0}\right\|=\left\|x_{0}\right\|$. By (3),

$$
\lim _{n \rightarrow \infty} f_{n}\left(x_{0}\right)=\left\|x_{0}\right\|=f_{0}\left(x_{0}\right) \text {. }
$$

By (1) and Corollary 2 of Theorem 2.1, we have $\lim _{m, n \rightarrow \infty}\left\|f_{m}-f_{n}\right\|=0$. Therefore, there exists $g_{0}$ in $\bar{B}$ with $\left\|g_{0}\right\|=1$ and $\lim _{n \rightarrow \infty}\left\|f_{n}-g_{0}\right\|=0$. Hence $\lim _{n \rightarrow \infty} f_{n}\left(x_{0}\right)=g_{0}\left(x_{0}\right)$ and $\lim _{n \rightarrow \infty} f_{n}\left(x_{0}\right)=f_{0}\left(x_{0}\right)$. Thus $g_{0}\left(x_{0}\right)=f_{0}\left(x_{0}\right)=\left\|f_{0}\right\|$ $=\left\|g_{0}\right\|=1$. From the remarks preceding this theorem we see that conditions (1) and (2) imply that $\bar{B}$ is strictly convex and so $f_{0}=g_{0}$. Therefore $\lim _{n \rightarrow \infty}\left\|f_{n}-f_{0}\right\|=0$. That is, $\bar{B}$ is 1.u.c. Q.E.D.

Combining Theorem 2.3 and Theorem 2.6 we have

THEOREM 2.7. If linear functionals attain their maximum on the unit sphere of $B$, then in order that $\bar{B}$ be l.u.c., it is n.a.s. that the norm be strongly differentiable in $B$, and $\bar{B}$ be weakly l.u.c. as a set of linear functionals. 
III. Spaces isomorphic with l.u.c. Banach spaces.

Definition 3.1. A sequence $\left\{x_{i}\right\}$ of elements of a Banach space $B$ is a basis for $B$ if, and only if, for every $x$ in $B$ there is a unique sequence of numbers $\left\{a_{i}\right\}$ such that $x=\sum_{i=1}^{\infty} a_{i} x_{i}$ in the sense that

$$
\lim _{n \rightarrow \infty}\left\|x-\sum_{i=1}^{n} a_{i} x_{i}\right\|=0 \text {. }
$$

If $\left\{x_{i}\right\}$ is a basis for $B$, then for some number $M>0$ and all $x$ in $B$

$$
\|x\| \geqq M \cdot \sup _{n}\left\|\sum_{i=1}^{n} a_{i} x_{i}\right\|, \quad \text { where } x=\sum_{i=1}^{\infty} a_{i} x_{i}[1, \mathrm{p} .111] .
$$

It follows from this that for every $x=\sum_{i=1}^{\infty} a_{i} x_{i}$ in $B,\left\{a_{i}\right\}$ is a bounded sequence, and $\sup _{i}\left|a_{i}\right| \leqq 2 \cdot\|x\| / M$.

Consider a basis $\left\{x_{i}\right\}$ with the property that for each number $c>0$ there exists a number $r_{c}>0$ such that

$$
\left\|\sum_{i=1}^{n} a_{i} x_{i}\right\|=1 \text { and }\left\|\sum_{i=n+1}^{\infty} a_{i} x_{i}\right\| \geqq c \text { imply }\left\|\sum_{i=1}^{\infty} a_{i} x_{i}\right\| \geqq 1+r_{c} .
$$

If $\left\{x_{i}\right\}$ is a basis for $B$ and $f$ is in $\bar{B}$, we denote by $\|f\|_{n}$ the norm of $f$ on $x_{n} \oplus x_{n+1} \oplus \cdots$ (the closure of the span of $\left(x_{n}, x_{n+1}, \cdots\right)$ ). A space $B$ with a basis will be said to have property $(\bar{A})$ if, for every $f$ in $\bar{B}, \lim _{n \rightarrow \infty}\|f\|_{n}=0$.

Condition (A) is satisfied by a basis $\left\{x_{i}\right\}$ if, and only if, $\sum_{i=1}^{\infty} a_{i} x_{i}$ converges whenever $\left\|\sum_{i=1}^{n} a_{i} x_{i}\right\|$ is bounded for all $n$. This condition and condition $(\overline{\mathrm{A}})$ constitute a necessary and sufficient condition for reflexivity [6, p. 519]. The space $c_{0}$ of sequences $a=\left\{a_{i}\right\}$ convergent to zero with $\|a\|$ $=\max _{i}\left|a_{i}\right|$ is a natural example of a space not satisfying (A), while $l_{1}$ does not have property $(\overline{\mathrm{A}})$. Clearly $l_{1}$ satisfies $(\mathrm{A})$, while it will be shown later that $c_{0}$ has property $(\overline{\mathrm{A}})$.

Theorem 3.1. If $B$ has a basis $\left\{x_{i}\right\}$ having property (A), then $B$ is isomorphic to a l.u.c. space.

Proof. Define a new norm \|\|$_{1}$, as follows: For $x=\sum_{i=1}^{\infty} a_{i} x_{i}$ in $B$, $\|x\|_{1}=\left(\|x\|^{2}+\sum_{i=1}^{\infty}\left(a_{i} / 2^{i}\right)^{2}\right)^{1 / 2}$, where \|\| is the old norm in $B$. It can be shown that there exists $R>0$ such that $\sum_{i=1}^{\infty}\left(a_{i} / 2^{i}\right)^{2} \leqq R^{2}\|x\|^{2}$ for all $x$. Therefore $\|x\| \leqq\|x\|_{1} \leqq\left(1+R^{2}\right)^{1 / 2} \cdot\|x\|$. So \|\|$_{1}$ is equivalent to \|\| . We now show that $B$ with norm \|\|$_{1}$ is l.u.c.

Let $x=\sum_{i=1}^{\infty} a_{i} x_{i},\|x\|_{1}=\left(\|x\|^{2}+\sum_{i=1}^{\infty}\left(a_{i} / 2^{i}\right)^{2}\right)^{1 / 2}=1$.

$$
y^{p}=\sum_{i=1}^{\infty} b_{i}^{p} x_{i}, \quad\left\|y^{p}\right\|_{1}=\left(\left\|y^{p}\right\|^{2}+\sum_{i=1}^{\infty}\left(\frac{b_{i}^{p}}{2^{i}}\right)^{2}\right)^{1 / 2}=1, \quad p=1,2,3, \cdots .
$$

Let $\lim _{p \rightarrow \infty}\left\|x+y^{p}\right\|_{1}=2$. We must show that

$$
\lim _{p \rightarrow \infty}\left\|x-y^{p}\right\|_{1}=0 \text {. }
$$


Suppose not. Then

$$
\left\|x-y^{p}\right\|_{1} \geqq s>0
$$

for a subsequence of $p$ 's. Using the fact that $\lim _{p \rightarrow \infty}\left\|x+y^{p}\right\|_{1}=2$, and an argument similar to the proof of Theorem 1.1, one can show that there exists a subsequence of $p$ 's such that $\lim _{p \rightarrow \infty}\left\|y^{p}\right\|=\|x\|$ and $\lim _{p \rightarrow \infty} b_{i}^{p}=a_{i}$, $i=1,2,3, \cdots$. It follows from this that

$$
\lim _{p \rightarrow \infty}\left\|x-: y^{p}\right\|=0 \text {. }
$$

For suppose not. Then there exists $t>0$ and a subsequence of $p$ 's such that

$$
\begin{aligned}
0<t<\left\|x-y^{p}\right\| & =\left\|\sum_{i=1}^{n}\left(a_{i}-b_{i}^{p}\right) x_{i}+\sum_{i=n+1}^{\infty} a_{i} x_{i}-\sum_{i=n+1}^{\infty} b_{i}^{p} x_{i}\right\| \\
& \leqq\left\|\sum_{i=1}^{n}\left(a_{i}-b_{i}^{p}\right) x_{i}\right\|+\left\|\sum_{i=n+1}^{\infty} a_{i} x_{i}\right\|+\left\|\sum_{i=n+1}^{\infty} b_{i}^{p} x_{i}\right\| \text { for each } n .
\end{aligned}
$$

Therefore $\left\|\sum_{i=n+1}^{\infty} b_{i}^{p} x_{i}\right\| \geqq t-\left(\left\|\sum_{i=1}^{n}\left(a_{i}-b_{i}^{p}\right) x_{i}\right\|+\left\|\sum_{i=n+1}^{\infty} a_{i} x_{i}\right\|\right)$. Hence

$$
\liminf _{p \rightarrow \infty}\left\|\sum_{i=n+1}^{\infty} b_{i}^{p} x_{i}\right\| \geqq t-\left\|\sum_{i=n+1}^{\infty} a_{i} x_{i}\right\| .
$$

Choose $n_{0}$ such that for all $n>n_{0}, t-\left\|\sum_{i=n+1}^{\infty} a_{i} x_{i}\right\| \geqq u>0$. Then for all $n>n_{0}$ we have $\lim \inf _{p \rightarrow \infty}\left\|\sum_{i=n+1}^{\infty} b_{i}^{p} x_{i}\right\| \geqq u>0$. Now $0<\|x\|<1$. Hence there exists $m_{0}$ such that for all $n>m_{0}, 0<\left\|\sum_{i=1}^{n} a_{i} x_{i}\right\|<1$. Therefore we have, for all $n>\max \left(n_{0}, m_{0}\right)$,

$$
\liminf _{p \rightarrow \infty} \frac{\left\|\sum_{i=n+1}^{\infty} b_{i}^{p} x_{i}\right\|}{\left\|\sum_{i=1}^{n} a_{i} x_{i}\right\|} \geqq \frac{u}{\left\|\sum_{i=1}^{n} a_{i} x_{i}\right\|}>u>0 .
$$

So for fixed $n>\max \left(n_{0}, m_{0}\right)$ there is a subsequence of $p$ 's such that

$$
\left\|\frac{\sum_{i=n+1}^{\infty} b_{i}^{p} x_{i}}{\left\|\sum_{i=1}^{n} a_{i} x_{i}\right\|}\right\| \geqq u \text { and }\left\|\frac{\sum_{i=1}^{n} b_{i}^{p} x_{i}}{\left\|\sum_{i=1}^{n} b_{i}^{p} x_{i}\right\|}\right\|=1 \text {. }
$$

By property (A) there exists $r_{u}>0$ such that

$$
\left\|\frac{\sum_{i=1}^{n} b_{i}^{p} x_{i}}{\left\|\sum_{i=1}^{n} b_{i}^{p} x_{i}\right\|}+\frac{\sum_{i=n+1}^{\infty} b_{i}^{p} x_{i}}{\left\|\sum_{i=1}^{n} a_{i} x_{i}\right\|}\right\| \geqq 1+r_{u} .
$$


Therefore

$\underset{p \rightarrow \infty}{\lim \inf }\left\|\frac{\sum_{i=1}^{n} b_{i}^{p} x_{i}}{\left\|\sum_{i=1}^{n} b_{i}^{p} x_{i}\right\|}+\frac{\sum_{i=n+1}^{\infty} b_{i}^{p} x_{i}}{\left\|\sum_{i=1}^{n} a_{i} x_{i}\right\|}\right\|=\frac{1}{\left\|\sum_{i=1}^{n} a_{i} x_{i}\right\|} \cdot \liminf _{p \rightarrow \infty}\left\|\sum_{i=1}^{\infty} b_{i}^{p} x_{i}\right\| \geqq 1+r_{u}$.

Thus $\lim \inf _{p \rightarrow \infty}\left\|y^{p}\right\| \geqq\left\|\sum_{i=1}^{n} a_{i} x_{i}\right\| \cdot\left(1+r_{u}\right)$. But $\lim _{\inf _{p \rightarrow \infty}}\left\|y^{p}\right\|=\lim _{p \rightarrow \infty}\left\|y^{p}\right\|$ $=\|x\|$ for every subsequence of $p$ 's. Hence $\|x\| \geqq\left\|\sum_{i=1}^{n} a_{i} x_{i}\right\| \cdot\left(1+r_{u}\right)$, and since $\lim _{n \rightarrow \infty}\left\|\sum_{i=1}^{n} a_{i} x_{i}\right\|=\|x\|$, we have $\|x\| \geqq\|x\| \cdot\left(1+r_{u}\right)>\|x\|$, which is a contradiction. Therefore $\lim _{p \rightarrow \infty}\left\|x-y^{p}\right\|=0$ in (3), and so $\lim _{p \rightarrow \infty}\left\|x-y^{p}\right\|_{1}=0$, which contradicts (2). Therefore $\lim _{p \rightarrow \infty}\left\|x-y^{p}\right\|_{1}=0$ in (1), and we have proved that $B$ with norm \|\|$_{1}$ is l.u.c. Q.E.D.

THEOREM 3.2. If $B$ has a basis $\left\{x_{i}\right\}$ and satisfies condition $(\overline{\mathrm{A}})$, then $B$ is isomorphic to a weakly l.u.c. space.

Proof. Define a new norm \|\|$_{1}$ as follows: For $x$ in $B, x=\sum_{i=1}^{\infty} a_{i} x_{i}$,

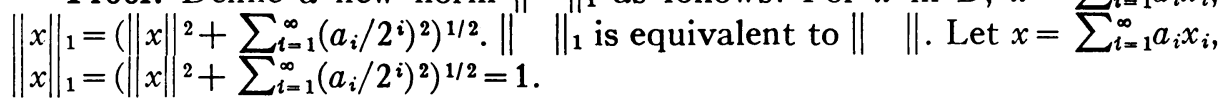

$$
y^{p}=\sum_{i=1}^{\infty} b_{i}^{p} x_{i}, \quad\left\|y^{p}\right\|_{1}=\left(\left\|y^{p}\right\|^{2} \sum_{i=1}^{\infty}\left(\frac{b_{i}{ }^{p}}{2^{i}}\right)^{2}\right)^{1 / 2}=1, \quad p=1,2, \cdots .
$$

Let $\lim _{p \rightarrow \infty}\left\|x+y^{p}\right\|_{1}=2$. We shall show that

$$
\lim _{p \rightarrow \infty} f\left(y^{p}\right)=f(x) \text { for every } f \text { in } \bar{B} \quad \text { [Definition 2.3]. }
$$

Suppose not. Then for some $f$ in $\bar{B}$ and some $r>0$, there is a sequence of $p$ 's for which

$$
\left|f\left(y^{p}\right)-f(x)\right| \geqq r>0 .
$$

As before, we can find a subsequence of this sequence for which $\lim _{p \rightarrow \infty}\left\|y^{p}\right\|$ $=\|x\|$ and $\lim _{p \rightarrow \infty} b_{i}^{p}=a_{i}, i=1,2, \cdots$. Now $\left\|y^{p}\right\|=\left\|\sum_{i=1}^{n} b_{i} x_{i}+\sum_{i=n+1}^{\infty} b_{i}^{p} x_{i}\right\|$ $\geqq\left\|\sum_{i=n+1}^{\infty} b_{i}^{p} x_{i}\right\|-\left\|\sum_{i=1}^{n} b_{i}^{p} x_{i}\right\|$, and so $\left\|\sum_{i=n+1}^{\infty} b_{i}^{p} x_{i}\right\| \leqq\left\|y^{p}\right\|+\left\|\sum_{i=1}^{n} b_{i}^{p} x_{i}\right\|$. Therefore,

$$
\underset{p \rightarrow \infty}{\limsup }\left\|\sum_{i=n+1}^{\infty} b_{i}^{p} x_{i}\right\| \leqq\|x\|+\left\|\sum_{i=1}^{n} a_{i} x_{i}\right\| .
$$

Now $f\left(y^{p}\right)=f\left(\sum_{i=1}^{n} b_{i}^{p} x_{i}\right)+f\left(\sum_{i=n+1}^{\infty} b_{i}^{p} x_{i}\right)$, and

$$
\left|f\left(\sum_{i=n+1}^{\infty} b_{i}^{p} x_{i}\right)\right| \leqq\|f\|_{n+1} \cdot\left\|\sum_{i=n+1}^{\infty} b_{i}^{p} x_{i}\right\| .
$$

Therefore, $\lim \sup _{p \rightarrow \infty}\left|f\left(\sum_{i=n+1}^{\infty} b_{i}^{p} x_{i}\right)\right| \leqq\|f\|_{n+1} \cdot\left(\|x\|+\left\|\sum_{i=1}^{n} a_{i} x_{i}\right\|\right)$. Hence 


$$
\begin{aligned}
\left|f\left(y^{p}\right)-f(x)\right| & =\left|f\left(y^{p}-x\right)\right| \\
& =\left|f\left(\sum_{i=1}^{n}\left(b_{i}^{p}-a_{i}\right) x_{i}\right)+f\left(\sum_{i=n+1}^{\infty} b_{i}^{p} x_{i}\right)-f\left(\sum_{i=n+1}^{\infty} a_{i} x_{i}\right)\right| \\
& \leqq\left|f\left(\sum_{i=1}^{n}\left(b_{i}^{p}-a_{i}\right) x_{i}\right)\right|+\left|f\left(\sum_{i=n+1}^{\infty} b_{i}^{p} x_{i}\right)\right|+\left|f\left(\sum_{i=n+1}^{\infty} a_{i} x_{i}\right)\right|
\end{aligned}
$$

and

$$
\underset{p \rightarrow \infty}{\lim \sup _{p}}\left|f\left(y^{p}\right)-f(x)\right| \leqq\|J\|_{n+1} \cdot\left(\|x\|+\left\|\sum_{i=1}^{n} a_{i} x_{i}\right\|\right)+\|f\|_{n+1} \cdot\left\|\sum_{i=n+1}^{\infty} a_{i} x_{i}\right\| .
$$

Since $n$ was arbitrary, and $\lim _{n \rightarrow \infty}\left\|\sum_{i=1}^{n} a_{i} x_{i}\right\|=\|x\|$, and $\lim _{n \rightarrow \infty}\left\|\sum_{i=n+1}^{\infty} a_{i} x_{i}\right\|$ $=0$, and, by our hypothesis, $\lim _{n \rightarrow \infty}\|f\|_{n+1}=0$, we have $\lim \sup _{p \rightarrow \infty} \mid f\left(y^{p}\right)$ $-f(x) \mid=0$. Therefore, $\lim _{p \rightarrow \infty}\left|f\left(y^{p}\right)-f(x)\right|=0$. But this contradicts (2). Therefore, (1) holds and the theorem is proved. Q.E.D.

An immediate consequence of Theorem 3.2 is that $c_{0}$ is isomorphic to a weakly 1.u.c. space. For $c_{0}$ has a basis (in fact the same basis that $l_{p}, p \geqq 1$, has $\left[1\right.$, p. 112]). And the adjoint space of $c_{0}$ is $l_{1}\left[1\right.$, p. 65]. Thus for $f$ in $l_{1}$, $f=\left\{C_{i}\right\}$, for some sequence of numbers, and $\|f\|=\sum_{i=1}^{\infty}\left|C_{i}\right|<\infty$. Thus $\lim _{n \rightarrow \infty}|| f \|_{n}=\lim _{n \rightarrow \infty} \sum_{i=n+1}^{\infty}\left|C_{i}\right|=0$. Moreover $c_{0}$ is not weakly l.u.c. To see this, consider the elements $x_{0}=(1,1,0,0, \cdots), y_{0}=(0,1,0,0, \cdots)$. Now for $x=\left\{a_{i}\right\}$ in $c_{0},\|x\|=\sup _{1 \leqq i<\infty}\left|a_{i}\right|$. So we have $\left\|x_{0}\right\|=1=\left\|y_{0}\right\|$ and $\left\|x_{0}+y_{0}\right\|$ $=2$. Consider the functional $f$ in $l_{1}$, where $f=(1,0,0, \cdots)$. Then $\|f\|=1$ $=f\left(x_{0}\right)$. But $f\left(y_{0}\right)=0$. And so $c_{0}$ is not weakly l.u.c. [Definition 2.3].

A basis $\left\{x_{i}\right\}$ is said to be unconditional if it has the property that whenever $x=\sum_{i=1}^{\infty} a_{i} x_{i}$, the series $\sum_{i=1}^{\infty} a_{i} x_{i}$ converges to $x$ for any rearrangement of the terms. It is known [6, p. 520] that a Banach space with an unconditional basis has property (A) unless it has a subspace isomorphic with $c_{0}$ and that it possesses property $(\overline{\mathrm{A}})$ unless it has a subspace isomorphic with $l_{1}$. Thus we have

THEOREM 3.3a. If $B$ has an unconditional basis and does not have a subspace isomorphic with $c_{0}$, then $B$ is isomorphic to a l.u.c. space.

Theorem 3.3b. If $B$ has an unconditional basis and does not have a subspace isomorphic with $l_{1}$, then $B$ is isomorphic to a weakly l.u.c. space.

We conclude with the remark that Clarkson [2, p. 412] showed that every separable normed space is isomorphic to a strictly convex space.

\section{BIBLIOGRAPHY}

1. S. Banach, Théorie des opérations linéaires, Warsaw, 1932.

2. J. A. Clarkson, Uniformly convex spaces, Trans. Amer. Math. Soc. vol. 40 (1936) pp. 396-414. 
3. M. M. Day, Reflexive Banach spaces not isomorphic to uniformly convex spaces, Bull. Amer. Math. Soc. vol. 47 (1941) pp. 313-317.

4. - Some more uniformly convex spaces, Bull. Amer. Math. Soc. vol. 47 (1941) pp. 504-507.

5. - Uniform convexity, Bull. Amer. Math. Soc. vol. 49 (1943) pp. 745-750.

6. R. C. James, Bases and reflexivity of Banach spaces, Ann. of Math. vol. 52 (1950) pp. 518-527.

7. V. Smulian, On some geometrical properties of the unit sphere in the space of the type $(B)$, Rec. Math. (Mat. Sbornik) N.S. vol. 48 (1939) pp. 90-94.

8. - Sur la derivabilite de la norme dans l'espace de Banach, C.R. (Doklady) Acad. Sci. URSS vol. 27 (1940) pp. 643-648.

University of California,

Berkeley, Calif. 